\title{
Inflammatory Cell Extraction and Nuclei Detection in Pap Smear Images
}

\author{
Dwiza Riana, Bandung Institute of Technology, Bandung, Indonesia \\ Marina E. Plissiti, Department of Computer Science and Engineering, University of Ioannina, \\ Ioannina, Greece
}

Christophoros Nikou, Department of Computer Science and Engineering, University of Ioannina, Ioannina, Greece

Dwi H. Widyantoro, Bandung Institute of Technology, Bandung, Indonesia

Tati Latifah R. Mengko, Bandung Institute of Technology, Bandung, Indonesia

Oemie Kalsoem, Veteran Bandung Laboratory of Pathology, Bandung, Indonesia

\begin{abstract}
The automated diagnosis of cervical cancer in Pap smear images is a difficult though extremely important procedure. In order to obtain reliable diagnostic information, the nuclei and their characteristics must be correctly identified and evaluated. However, the presence of inflammatory and overlapping cells in these images complicates the detection process. In this work, a segmentation algorithm is developed to extract the inflammatory cells and enable accurate nuclei detection. The proposed algorithm is based on the combination of gray level thresholding and the definition of a distance rule, which entails in the identification of inflammatory cells. The results indicate that our method significantly simplifies the nuclei detection process, as it reduces the number of inflammatory cells that may interfere.
\end{abstract}

Keywords: Cytoplasm Segmentation, Inflammatory Cells, Nucleus Detection, Pap Smear Images, Thresholding

\section{INTRODUCTION}

Cervical cancer is one of the most common threats for women worldwide, as it has been the second highest cause of cancer-caused deaths among women (Smith et al, 2014). Pap smear examination is one of the most common methods used to detect cervical cancer. The specific examination is a preventative measure to detect the presence of pre-cancerous and cancerous situations in cervical cell samples. The diagnostic value of the Pap smear is verified by the 
Figure 1. Conventional Pap smear cell images with inflammatory cells
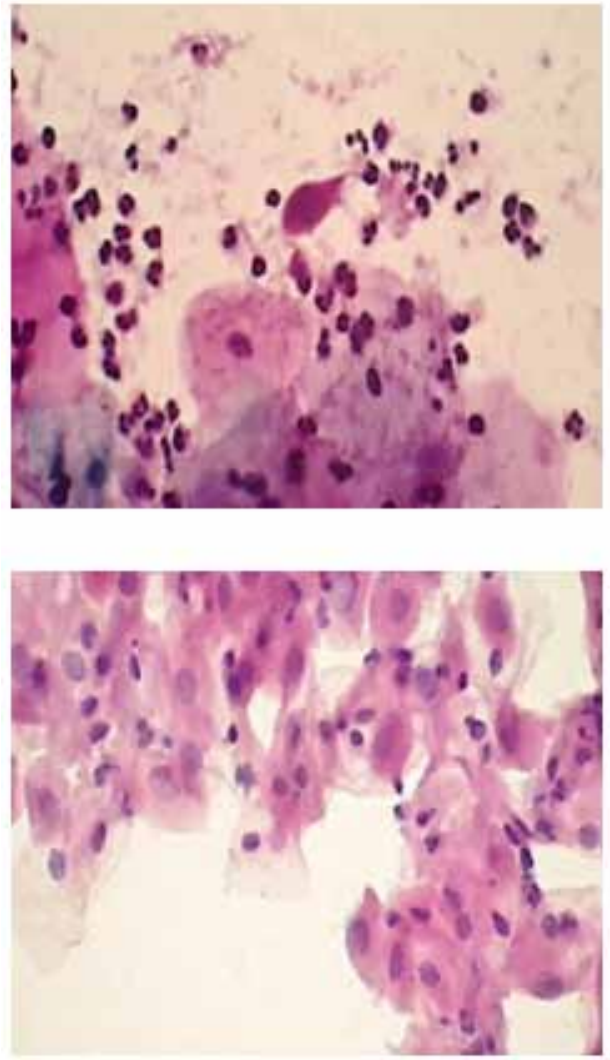

fact that cervical cancer incidence and mortality rates have declined since the introduction of the Pap smear test in the mid-20th century (Papanicolaou, 1942), and the rates continue to decline till now (Smith et al, 2014).

Pap smear images present particular characteristics, and their automated interpretation is still a challenging issue for the researchers. The diversity of the cell structures, the intense fluctuation of the background and the variances in illumination and dye concentration of the cells due to the staining procedure are some of the most representative limitations that any automated method should effectively deal with.

The presence of inflammatory cells and blood in the background is a common feature of conventional Pap smear images. In Figure 1 some examples of the inflammatory and over-
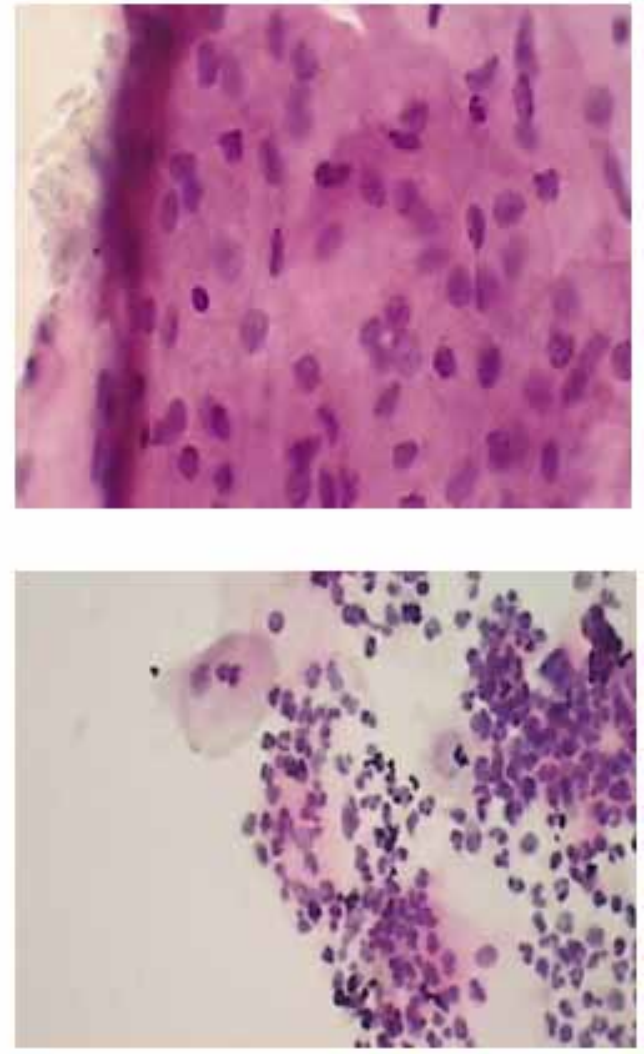

lapping cells are depicted. From these examples it is clear that an image analysis technique should be firstly performed in order to achieve a reliable interpretation about the contents of each image. This technique could include a number of steps, such as a) inflammatory cell extraction, b) segmentation of normal cells and abnormal cells, c) identification of menopause cells due to the increasing level of hormone, d) background cleaning due to the existence of blood cells. This work is focused on the extraction of inflammatory cells.

Although there are plenty of methods proposed in the literature for the analysis of Pap smear images, the problem of inflammatory cell identification has not been addressed yet. More specifically, the determination of the nucleus and cytoplasm borders in cervical images that 
contain only one cell or isolated cells has been considered by several researchers (Bamford et al, 1996, Bamford et al, 1998, Lassouaoui et al, 2003, Yang-Mao et al, 2008, Lin et al, 2009). The methods proposed in (Plissiti et al, 2011(a), Plissiti et al, 2011(b)) deal with the detection of nuclei locations and the nuclei boundary delineation respectively, in conventional Papstained cervical cell images which may contain both isolated cells and cell clusters.

Furthermore, many methods do not directly use the color information of cervical images. In (Garrido et al., 2000) a method using grayscale images which affects the excess of edge points or overlapped objects in complex images was proposed. In addition, genetic algorithms (Lassouaoui et al, 2003), pixel classification (Baak et al, 2004), region growing (Mat Isa, 2005) and deformable models (Plissiti et al., 2010) contour detectors (Tsai et al, 2008; Malm \& Brun, 2009) were also proposed for the segmentation of cervical images using grayscale images. It must be noted that neither of the above mentioned studies deal with the existence of inflammatory cells in Pap smear images.

Segmentation of cells images by thresholding has been proposed by several researchers (Poulsen \& Pedron, 1995; Wu, Gil, \& Barba, 1998; Plissiti, \& Nikou, 2013; Riana, Dewi, Widyantoro \& Mengko, 2014). Through this technique, the binary images that are extracted by thresholding the initial image are clearly simpler to analyze and significantly reduce the amount of data. Besides, the variance based thresholding methods are used extensively for image segmentation (Hou, Hu, \& Nowinsk, 2006; Tsai, Chan, Lin, Yang-Mao, \& Huang, 2010). In general, the objective of these methods was to automatically identify threshold values in order to separate the cell from the background and the nucleus from the cytoplasm. A comparison of some threshold selection methods is performed by (Sezgin, \& Sanlur, 2004). A thresholding technique was also proposed regarding a multiscale local adaptive threshold method based on shape stability on the extraction of the nuclei region from the background (Li et al, 2007).
In this paper, we propose a methodology for cervical cell image analysis, which aims at two specific goals: a) the detection of nuclei and cytoplasm locations and b) the extraction of inflammatory cells in images containing isolated cells. Thus, the main contribution of our paper is twofold. Firstly, the parts of the image that do not include helpful findings, such as the background, are discarded. Secondly, the extraction of inflammatory cells leads to the isolation of the true nucleus of each cell, providing additional and complementary information. By these means, the expert cytologist may obtain a reliable diagnostic decision about the Pap smear slide. The method is based on thresholding techniques and provides effective image segmentation. The method incorporates a priori knowledge about the position of the nuclei, whose estimation relies on the centroid of the cytoplasm. In general, the cell nucleus is located in the centre of the surrounding cytoplasm. Based on this fact, we propose a method that can distinguish the nuclei locations in Pap smear images. The method exploits the particular nuclei and cytoplasm characteristics through morphological image analysis. Finally, the method is evaluated using a test set of 222 cytological images of conventional Pap smears, which contain in total 418 isolated cells surrounding by inflammatory cells. The remainder of this paper is organized as follows: section 2 describes in detail the proposed methodology. In section 3, the results of the method applied to our image data base are presented and in section 4 some interesting conclusions about the performance of the method and the processed images are discussed.

\section{MATERIALS AND METHODS}

In this work we have used several Pap smear images which contain single or overlapping cells surrounded by inflammatory cells. These images were obtained through microscopic slides using a digital camera adopted on a microscope. The images were stored in JPEG format. We have constructed an image database that is 
Figure 2. Examples of original images

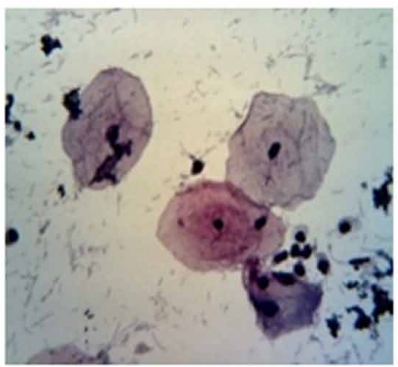

(a)

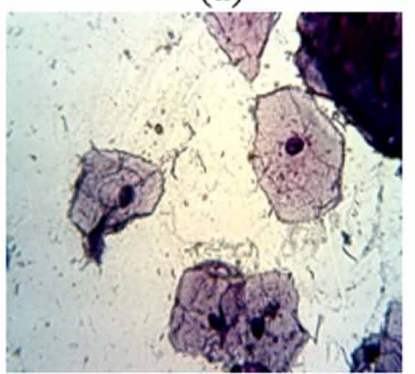

(d)

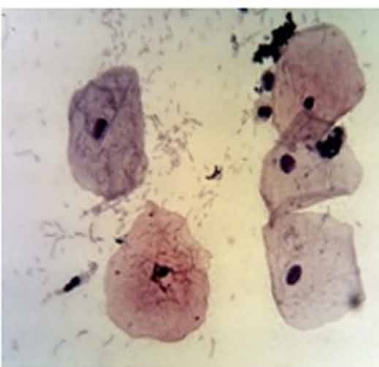

(b)

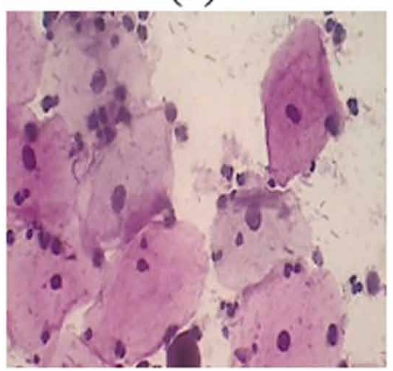

(e)

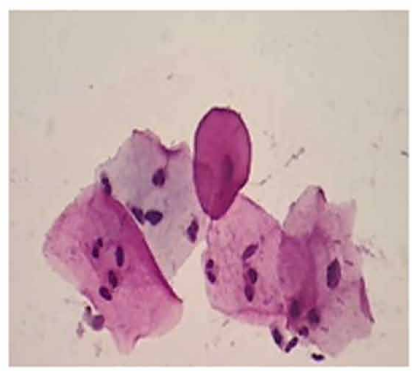

(c)

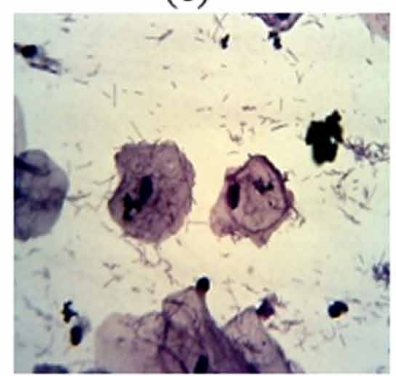

(f) based on the laboratory observation results given by a pathologist in Clinical Pathology Laboratory in Indonesia. The database consists of 222 images in total. Figure 2 (a-f) depicts some examples of the images in this work. The characteristics of cells in all images are normal cells with inflammatory cells, except for (2e) that is characterized as normal/abnormal cell (atipic criteria).

A block diagram of the proposed method is depicted in Figure 3. The first stage is the pre-processing step which includes color conversion, adjustment and filtering of the image in order to prepare it for further processing. The next step is the processing of the enhanced image, whose objective is to segment a single cell from the background and to extract its nucleus from the inflammatory cells. Next, the feature extraction process is applied, in which some salient features are computed, such as the area, the perimeter and the roundness of the nucleus and cytoplasm. These features are extracted for further analysis. Each stage is discussed in details in the next paragraphs.

\subsection{Prepocessing}

The pre-processing stage enhances the quality of the images for further processing. Pap smear images are colored images of poor quality due to the stain variations that are used to color the cells, and the uneven lighting across the field of view. The color information is insignificant compared to the intensity of the images. Hence in the pre-processing stage, the first step is to convert the RGB image to Intensity/Gray scale image. The grayscale value was obtained by forming a weighted sum of the R, G, and B components as given in (1).

$$
I_{\text {gray }}=0.2989 * R+0.5870 * G+0.1140 * B
$$

A well-defined boundary between the nucleus and the cytoplasm is required to extract 
Figure 3. Scheme of the proposed method

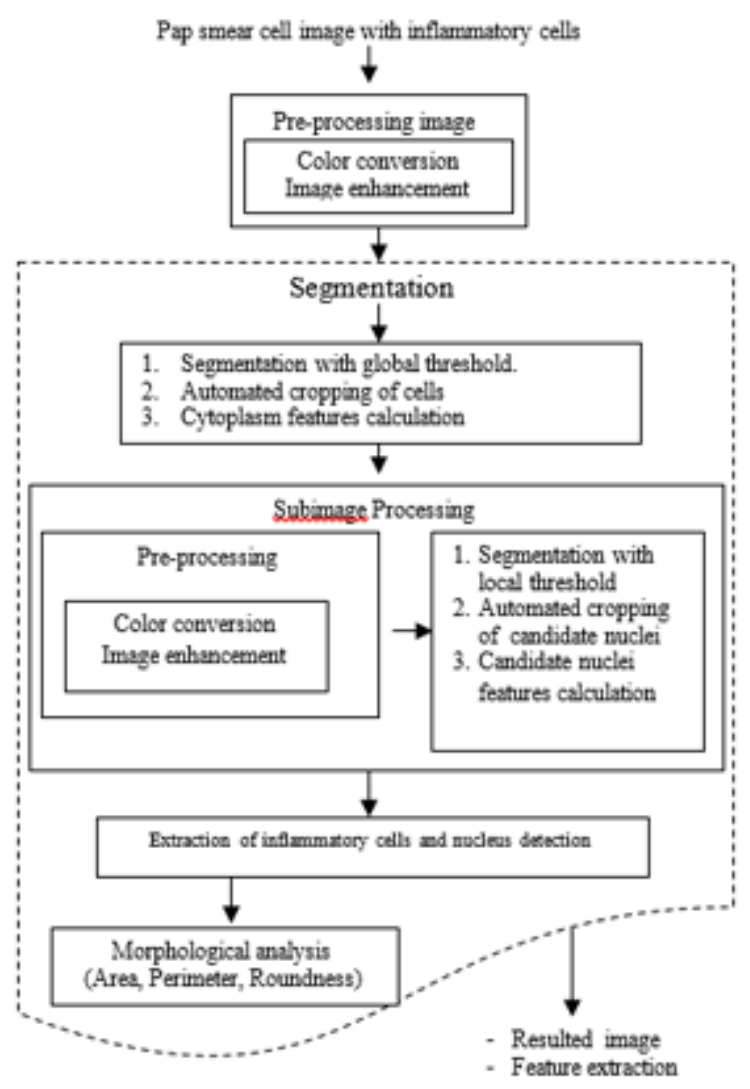

the nucleus from the cell. However, the contrast of the Pap smear images is poor and the lack of homogeneity in the image intensity makes the further processing difficult. Thus, it is necessary to improve the contrast of the image in order to get clear edges and boundaries of the nucleus. This is a prerequisite for the accurate segmentation of the image and the reliable feature extraction.

Image adjustment and filtering are used to enhance the contrast of the image, resulting in cell boundaries that are clearly distinguishable from the background of the image. Thus the quality of the image is improved, making it more suitable for the application of the segmentation procedure. With this procedure, the intensity values in the low-contrast grayscale image are mapped to new values in the adjusted image so that $1 \%$ of the data is saturated at low and high intensities of the grayscale image. Thus, the contrast of the output image is increased. Unsharp masking is used in the image sharpening step, which results in an edge image $g(x, y)$ from an input image $f(x, y)$ given by:

$g(x, y)=f(x, y)-f_{\text {smooth }}(x, y)$

where $f_{\text {smooth }}(x, y)$ is a smoothed version of $f(x$, $y)$. The sharpened image is given by:

$f_{\text {sharp }}(x, y)=f(x, y)+k * g(x, y)$ 
Figure 4. The original image and the results of the preprocessing step

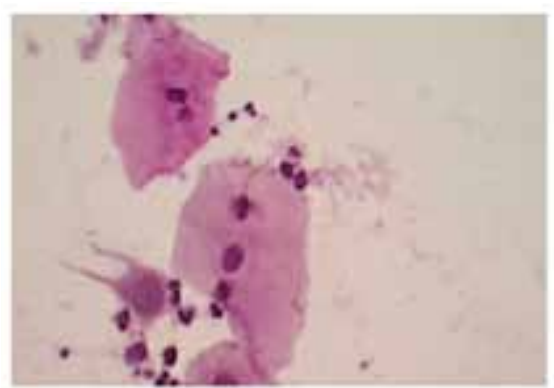

(a) Original image

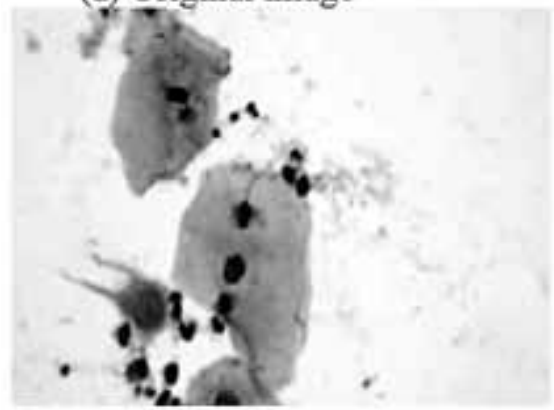

(c) Adjusted image

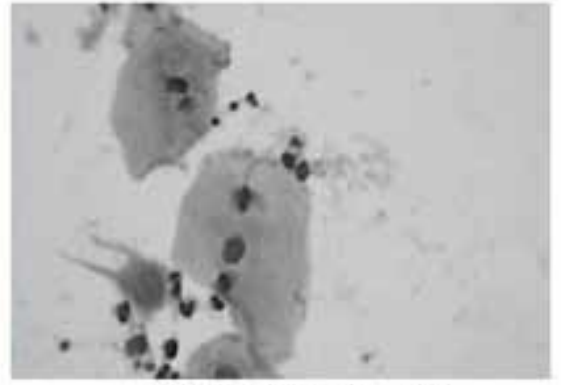

(b) Grayscale image

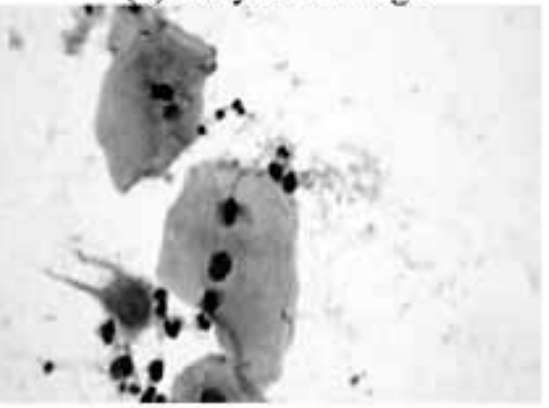

(d) Sharpened image with $k$ varying between 0.2 and 0.7 .

As it can be seen in Figure 4(d), after the preprocessing step the image is clearer than the initial image, as its edges and other high frequency components have been enhanced. The image obtained from the preprocessing step is then used as input in the next step for the extraction of the inflammatory cells.

\subsection{Segmentation}

In this step, the image is processed in order to effectively detect the regions of interest (in our case the cells) from the background. The background in the image is expected to exhibit homogeneous characteristics, as the noise is removed in a high degree in the preprocessing step. Furthermore, the background presents significant difference from the areas of the cells, because in most cases the intensity of the cells is lower than the intensity of the background. For these reasons, image thresholding provides an easy and effective way to define the regions of interest in the specific images.

The results of the thresholding process are highly depended on the choice of the threshold value. In our experiments, we specify the value of the threshold to 0.65 for all the images in our data set. This value has been empirically obtained by trial and error for the 222 input images.

After the application of the thresholding process in the images, we extract binary images containing the regions of interest, which are depicted as the white areas constructed by 4-connected components. These areas are expected to be the isolated cells or the cell clusters of the image. Thus, some auxiliary features of these objects are calculated, such as the area and the centroid, which will be used later for the definition of the nuclei of the cells, as it is explained in the next paragraphs.

In order to take advantage of the local characteristics of the image and to indepen- 
Figure 5. Illustration of the centroid and the bounding box of the detected objects in the image. The region consists of the white pixels; the green box is the bounding box, and the red dot is the centroid

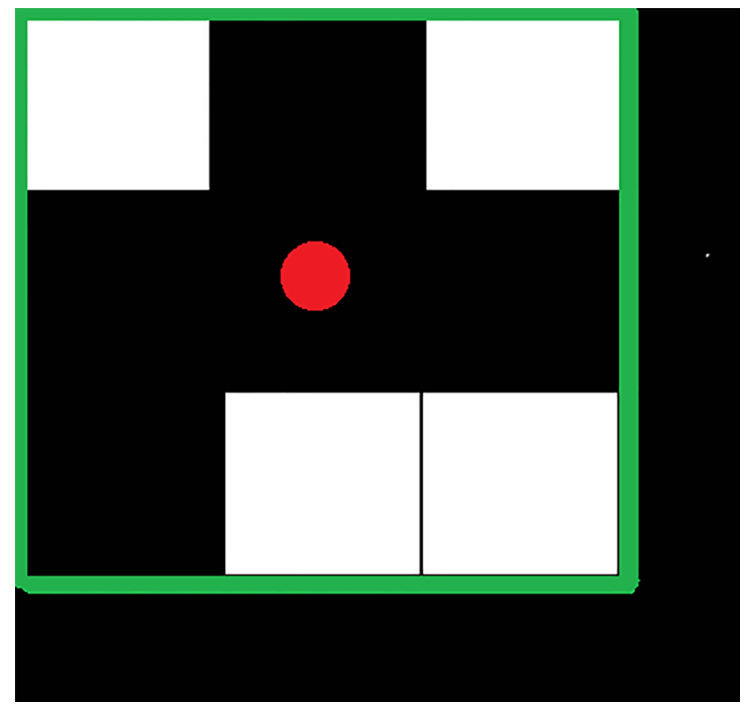

Figure 6. Results of the automated cropping and the definition of subimages

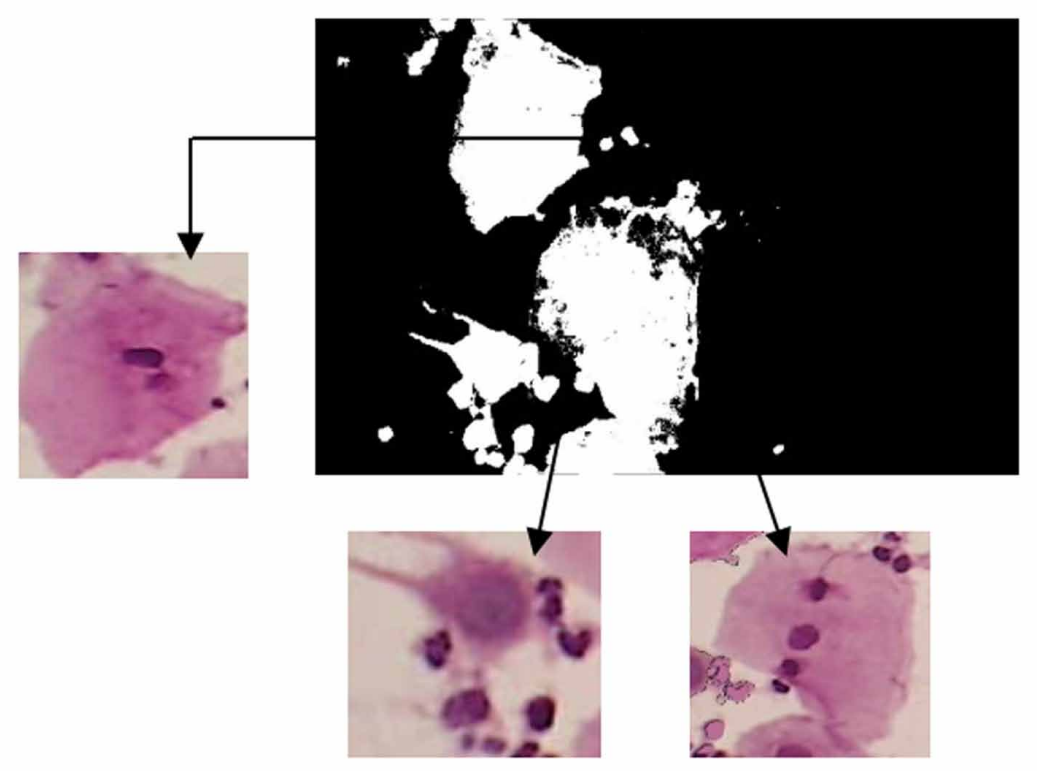


Figure 7. Result of the preprocessing step in the obtained subimages
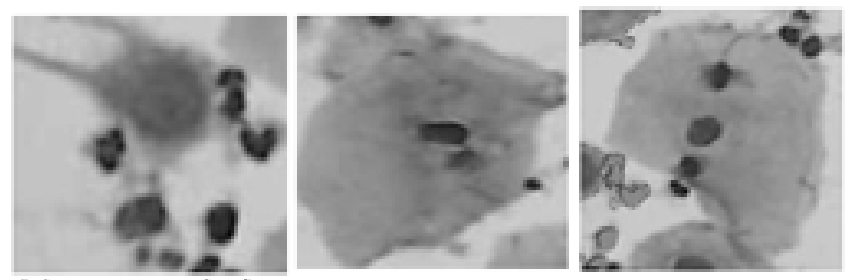

(a) Grayscale images

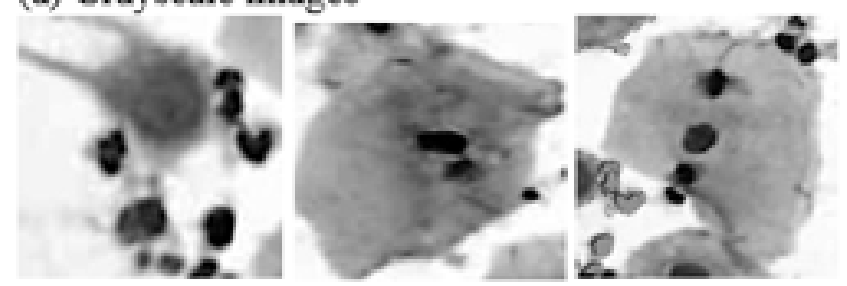

(b) Adjusted images

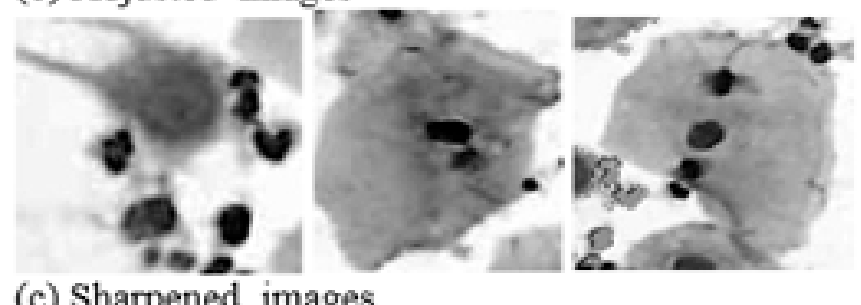

(c) Sharpened images

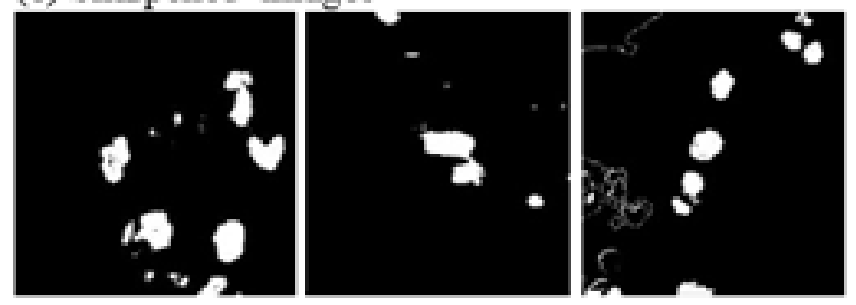

(d) Binary images

dently process each detected cell we proceed with the automated cropping of the image. For this reason we have used the smallest rectangle (bounding box) containing the region. Figure 5 illustrates the centroid and bounding box of an image. The automated cropping process is repeated according to the number of cells in the original images (Figure 6) resulting in a set of subimages.

For each extracted subimage, we follow a preprocessing step, similar to the aforemen- tioned procedure in the previous paragraph (grayscale image adjustment and filtering) for the enhancement of the local image characteristics. Furthermore, a thresholding procedure is then performed in each subimage, for the extraction of the candidate nuclei in the cell. This is based on the fact that the nuclei area is darker than the cytoplasm area in each subimage. The threshold value is set to 0.25 for all the subimages resulted by the automated cropping 
Figure 8. The candidate nuclei of the cell
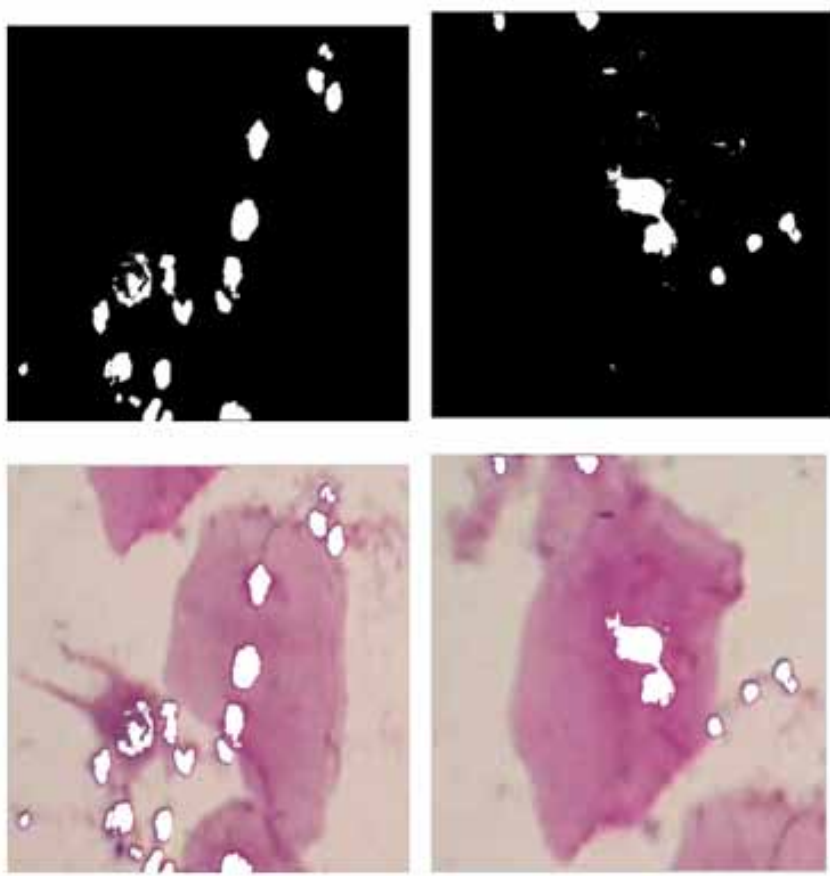

of the 222 original images in our data set. This value has been obtained empirically.

In Figure 7 we can see the several steps of the thresholding procedure in three subimages. As we can observe, in Fig. 7(d) the resulted binary images may contain more than one candidate nucleus. However, as we expect in each subimage to detect only one cell (Figure 8 ), and consequently only one nucleus, further processing is necessary in order to define the true nucleus in each subimage and to extract the inflammatory cells.

\subsection{Nuclei Definition and Extraction of Inflammatory Cells}

The detection of inflammatory cells is based on a priori knowledge about the geometrical features of the cell. In our work, we considered that the shape of the cell follows an elliptical pattern and the position of the nucleus in the cell is assumed to be close to the centroid of the cytoplasm. Thus, a distance rule is defined, comparing the Euclidean distance of the centroid position of all the candidate nuclei and the centroid of the cytoplasm.

The definition of the shortest distance between a nucleus and the centroid of the cytoplasm indicates the existence of the true nucleus. All the other candidates which have larger distances from the cytoplasm centroid are discarded, and they are characterized as inflammatory cells. These inflammatory cells will be extracted and eliminated by changing their color into a color similar to the color of the cytoplasm. The detected cell nucleus is remaining with its original color. Figure 9(b) shows the result of this procedure in an original image. In this figure, we can see that the inflammatory cells in this image are suppressed. 
Figure 9. Extraction of inflammatory cells in an image after the application of our method

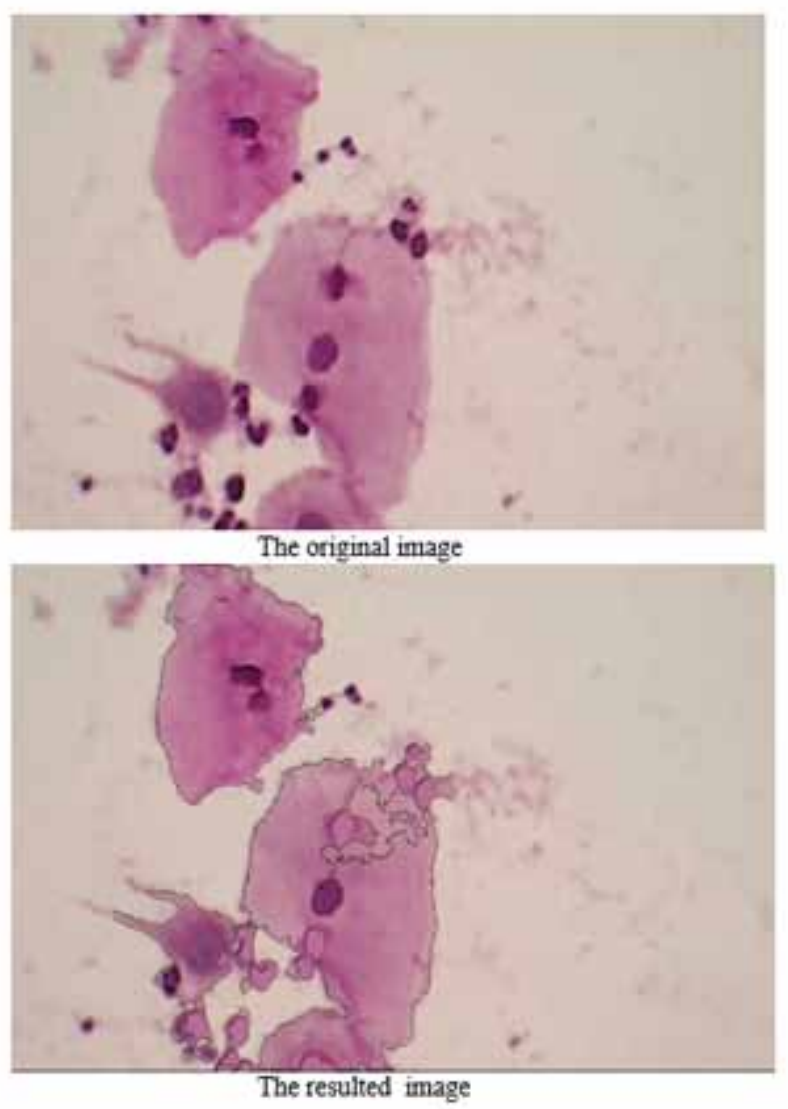

\subsection{Morphological Feature Extraction for the Cytoplasm and the Nucleus}

The next step is to perform morphological analysis of the shape of the regions of interest in the resulted images in order to extract useful conclusions for their contents. Morphological feature extraction is performed, by calculating the area, the perimeter and the roundness of the region of interest. Nucleus area is calculated from the set of white pixels for each detected nuclei in the bounding box. Similarly, the cytoplasm area is calculated by counting the white pixels in the bounding box of the cytoplasm.
Perimeter is the number of the pixels consisting the object boundary. Finally, for the calculation of the roundness we have used the following formula (6):

Roundness $=4 \pi$ Area $/$ Perimeter $^{2}$

An example of the morphological feature extraction process in real images can be seen in Table I. The analysis of such morphological cell features is one of the most important processes, in order to derive reliable diagnostic conclusions and to early detect cervical cancer. 
Table 1. The results of feature morphology

\begin{tabular}{|c|c|c|c|c|}
\hline Properties & Cytoplasm & Nucleus & Cytoplasm & Nucleus \\
\hline Area & 65447 & 1534 & 108865 & 3271 \\
\hline Perimeter & 2441.454 & 183.4386 & 2188.542 & 236.4508 \\
\hline Roundness & 2740.324 & 0.134742 & 4123.69 & 0.277703 \\
\hline
\end{tabular}

\section{RESULT}

In our experiments, we used 222 cytological images of conventional Pap smears, from which we automatically obtained 418 cropped images (255 images of single or isolated cells and 163 images of overlapping cells). For the construction of the training set we have selected 143 out of the 222 aforementioned images. Consequently, we came out with 293 cropped images (169 images of isolated cells and 124 images of overlapping cells). These images are used for the estimation of the threshold values.

The proposed method was tested in terms of its accuracy on the determination of the nuclei and cytoplasm boundaries, using a test set of 79 images with inflammatory cells, which contained 125 cropped images (86 images of isolated cells and 39 images of overlapping cells). It must be noted that the training and the test set of the images were independent. These images were acquired through a Logitech camera (Logitech HD C525) adapted to an optical microscope (Olympus CH20). We have used a 40x magnification lens and the acquired images were stored in JPEG format. The images were then cropped automatically in order to capture a single cell containing the inflammatory cells.

\subsection{Extraction of Inflammatory Cells}

The application of our method to the aforementioned image data set produced promising results, which indicate that the method is able to detect inflammatory cells and to define accurately the cytoplasm and the nucleus (Figure 10). This was achieved in 86 single cell images, where the inflammatory cells and the segmentation of the cytoplasm and nucleus were successfully extracted. In these images 39 inflammatory cells were correctly identified, which were then colored similar to the cytoplasm color.

\subsection{Morphological Feature Analysis}

We evaluated 86 images for the extraction of inflammatory cells and successfully segment cytoplasm and nucleus. In these images, we have calculated three morphological features of the cytoplasm and the nucleus, which are the area, the perimeter and the roundness.

In Figures 11 and 12, we can see the results of the comparison of the area and the perimeter of the nucleus and the cytoplasm respectively. As it was expected, the cytoplasm has a bigger area and perimeter than the nucleus. 
Figure 10. Results of the proposed method for the extraction of inflammatory cells and the detection of the cytoplasm and nucleus
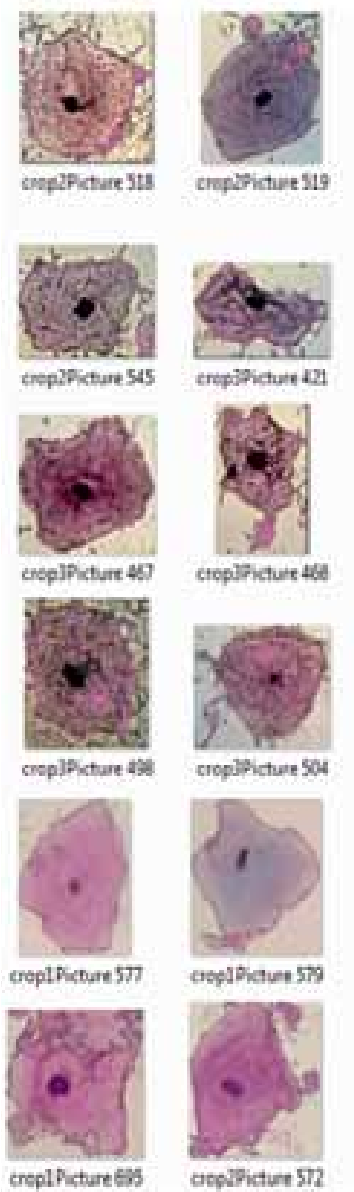

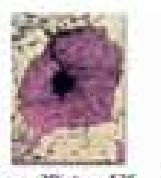

copepichare 506

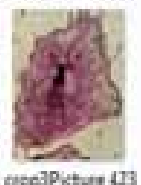

oseapisture a3
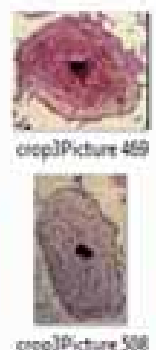

cosplpicture Ste

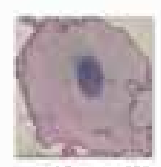

eeptipiantest

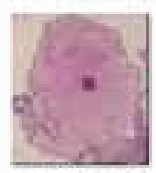

crep2Picture $5 \pi$
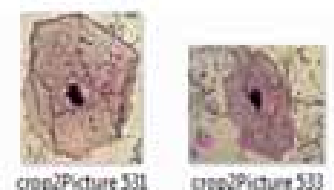

anolpicture 513

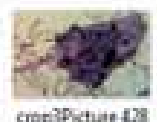

crepipictuate 4?
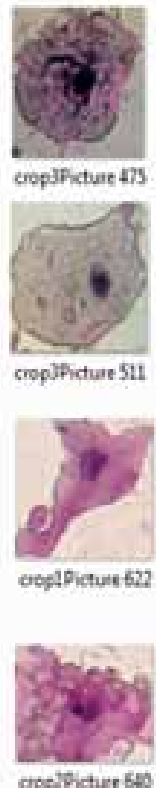
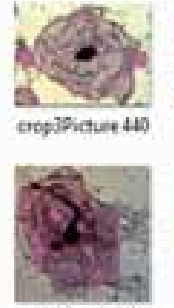

crepipictunt 484

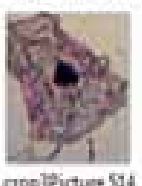

cooploicture SY4
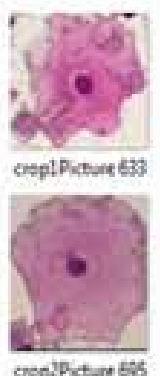
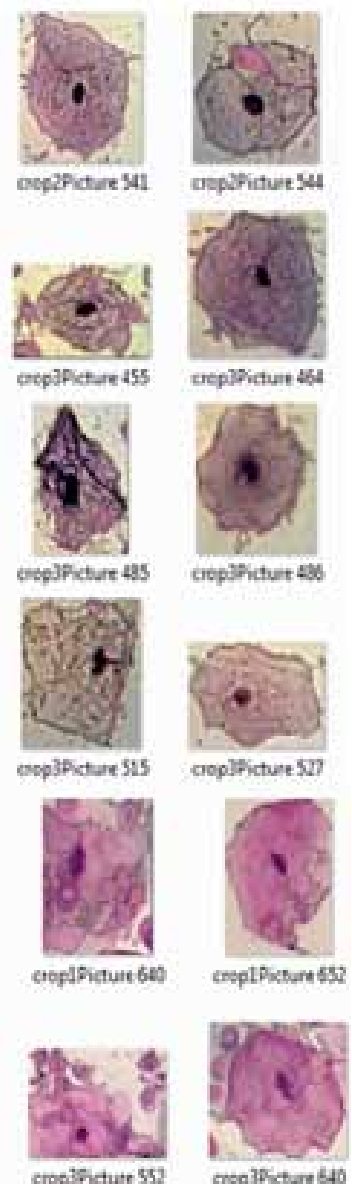

Table 2 shows the measured features of the cytoplasm and the nucleus produced be our method in the 86 images, in terms of mean \pm standard deviation. Thus the result for the cytoplasm area was $122741.9 \pm 40075.61$, whereas the corresponding result for the nucleus was $7218.55 \pm 9101$. The high value of the standard deviation of the cytoplasm area can be explained as the cytoplasm intensity values exhibit extent variation compared to the intensity values of the nucleus.

Figure 13 depict the values of the roundness of each cytoplasm and nucleus. As we can observe, the nuclei of all cells tent to have round shapes; this is also shown by the result of the roundness, which is $0.179 \pm 0.311$. It points out that the normal nuclei usually have a smooth and circular shape. On the contrary, cytoplasm has various shapes, which result in the cytoplasm roundness of $14455.92 \pm 51482.56$.

\section{DISCUSSION}

In this study we proposed a method for the extraction of inflammatory cells and the suc- 
Figure 11. Comparison between the cytoplasm (upper curve) and nucleus (lower curve) areas in 86 images

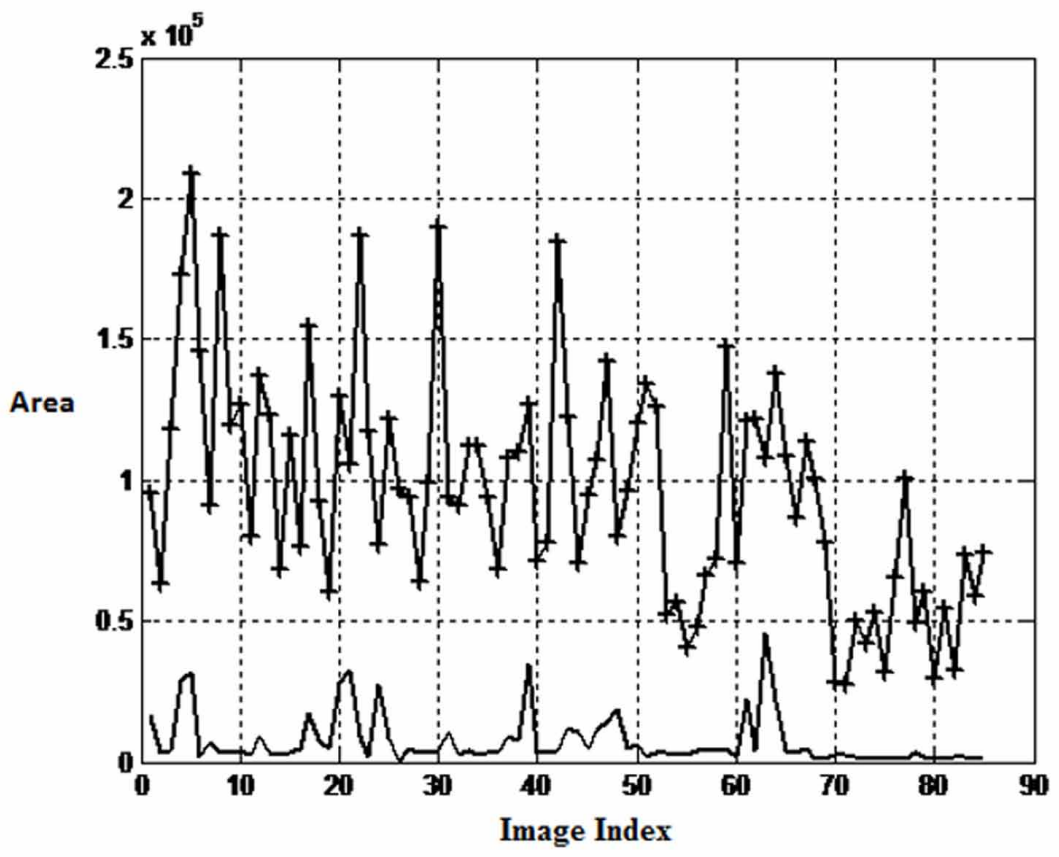

Figure 12. Comparison between the cytoplasm ("+”) and the nucleus (continuous line) perimeters in 86 images

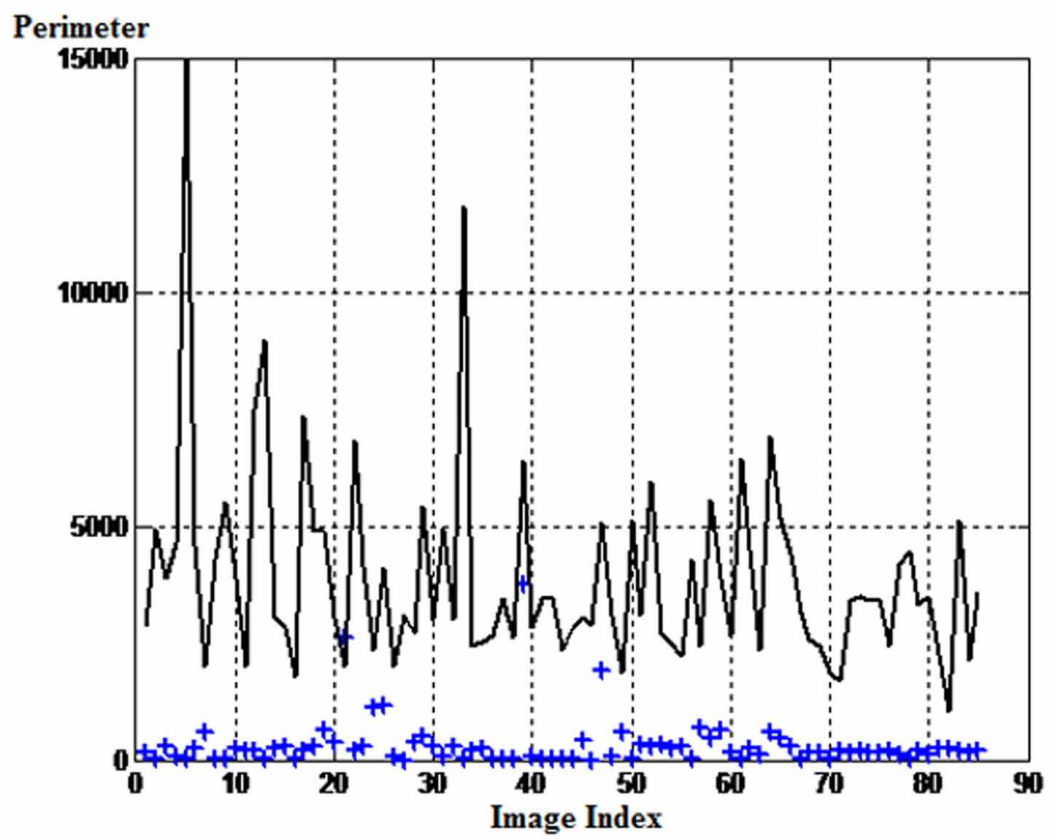


Table 2. Morphological features of nucleus and cytoplasm

\begin{tabular}{|l|l|l|l|l|}
\hline \multicolumn{1}{|c|}{ Cell } & \multicolumn{1}{c|}{ Feature } & \multicolumn{1}{c|}{ N } & \multicolumn{1}{c|}{ Mean } & \multicolumn{1}{c|}{ St. Dev } \\
\hline \multirow{4}{*}{ Cytoplasm } & Area & 86 & 122741.9 & 40075.61 \\
\cline { 2 - 5 } & Perimeter & 86 & 4326.79 & 3718.47 \\
\cline { 2 - 6 } & Roundness & 86 & 14455.92 & 51482.56 \\
\hline \multirow{3}{*}{ Nucleus } & Area & 86 & 7218.55 & 9101 \\
\cline { 2 - 5 } & Perimeter & 86 & 413.28 & 797.67 \\
\cline { 2 - 5 } & Roundness & 86 & 0.179 & 0.311 \\
\hline
\end{tabular}

cessful detection of cell nuclei in Pap smear images. Tests on 79 images containing 125 cells were performed and the method produced accurate results in a total number of 86 single cell images, in which the identification of the cytoplasm and the nucleus was reliable. In the other 39 cell images, the method successfully extracted the inflammatory cells and detected the cytoplasm boundary, but it missed to define the nuclei of each overlapping cell.
As it was verified by an expert observer (in our case a pathologist), the method leads to the perfect detection of the 86 cells in the corresponding images of our data set. These images contained normal superficial epithelial cells which tent to exhibit circular shape and their nucleus are located in the center of the cytoplasm area (Figure 10). However, in the remaining 39 images (Figure 14) in which the method missed to identify some nuclei, we can observe that either the cytoplasm bound-

Figure 13. Comparison of the roundness of the cytoplasm and the nucleus in 86 images

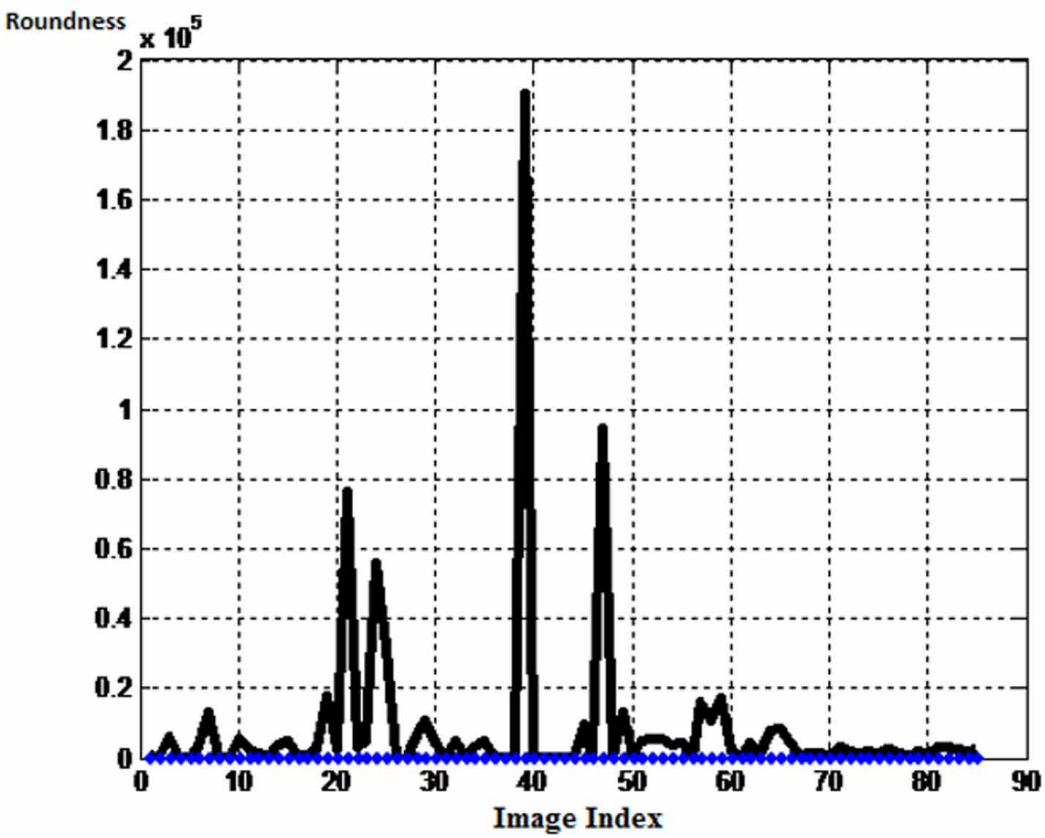

Copyright $($ C 2015, IGI Global. Copying or distributing in print or electronic forms without written permission of IGI Global is prohibited. 
Figure 14. Application of the proposed method in images with overlapping cells. (a), (c) Original images, (b), (d) resulted images. It must be noted that although the method requires further improvement, it achieves to accurately define the cytoplasm area

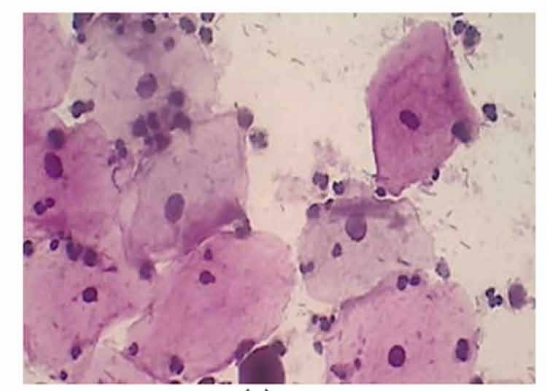

(a)

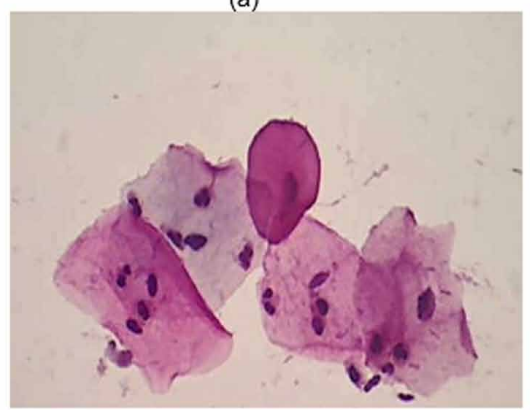

(c)

ary is not correctly delineated, either there are overlapping cells.

In this work, we have also performed morphological feature analysis of the detected cells. From the mean and the standard deviations of all of the results of the morphological analysis, there is a clear difference between the average size and the standard deviation of cytoplasm and nucleus. This can be explained as the images included in our data set (125 images) contained only normal superficial cells, which exhibit a large ratio of cytoplasm and nucleus. This is consistent with the calculated area, perimeter and roundness, which showed normal superficial cells.

The results indicate that the proposed method has successfully detected the nucleus and cytoplasm of the isolated cells in Pap smear images, especially cells under normal conditions (normal superficial class) providing that

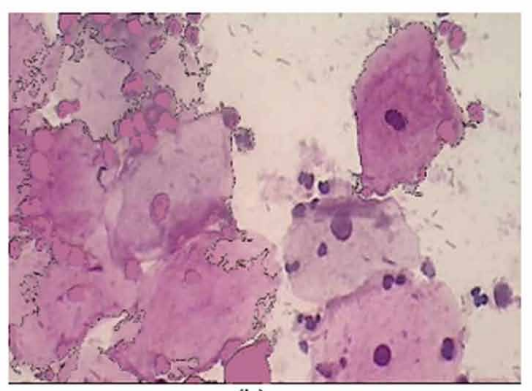

(b)

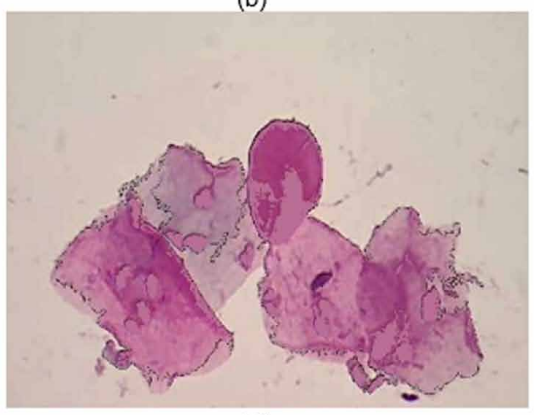

(d)

the cytoplasm is not folded or overlapped. The identification of the cervical cell nuclei areas in the conventional Pap smear images remains a difficult problem, especially in overlapping cells and those presented inflammation. The accurate segmentation of the area of the nucleus is a prerequisite for the derivation of the diagnostic conclusions and the characterization of the contents in the Pap smear images.

However, in some images, the results were not as accurate as we expected to be. More specifically, although the proposed method successfully identified the inflammatory cells and their cytoplasm, their nucleus was not accurately detected. The reason for these results was the overlapping of cells, as it can be seen in Figure 14. As we can see in this image, the results of our method in overlapping cells lead in the erroneous detection of the nucleus of each cell. This result is obtained as we consider that 
the cytoplasm area that was detected contained only one cell, and for this reason the method detects only one nucleus corresponding to the specific cytoplasm area.

\section{CONCLUSION}

In this work, we proposed an automated methodology for the extraction of inflammatory cells in Pap smear images. This task is particularly useful for the correct interpretation of microscopic images and the derivation of accurate diagnostic conclusions. We can come out with the fact that the proposed method is suitable for the extraction of the inflammatory cell in the normal superficial class. However, we need more reliable practical alternative methods to segment Pap smear images effectively. As future work, we intend to extend our method in order to be able to accurately recognize overlapping cells and improve its performance. Furthermore, we consider extracting inflammatory cells that overlap in normal or abnormal Pap smear cell images.

\section{ACKNOWLEDGMENT}

Dwiza Riana would like to thank Directorate General of Higher Education-Ministry of Education and Culture, (DIKTI), Indonesia through BPPS (2011), DGHE Sandwich Program(2013) and Doctoral Dissertation Research (2014) for generous scholarship, Laboratorium Patologi Veteran Bandung, Indonesia for the database Pap smear images and University of Ioannina, Greece for location of DGHE Sandwich Program.

\section{REFERENCES}

Bak, E., Najarian, K., \& Brockway, J. P. (2004). Efficient segmentation framework of cell images in noise environments. In Proc. 26th Int. Conf. IEEE Eng. Med. Biol., vol. 1, (pp. 1802-1805). doi:10.1109/ IEMBS.2004.1403538
Bamford, P., \& Lovell, B. (1996). A water immersion algorithm for cytological image segmentation. In Proc. APRS Image Segmentation Workshop, Sydney, Australia, (pp. 75-79).

Bamford, P., \& Lovell, B. (1998). Unsupervised cell nucleus segmentation with active contours. Signal Processing, 71(2), 203-213. doi:10.1016/S01651684(98)00145-5

Garrido, A., \& de la Blanca, N. P. (2000). Applying deformable templates for cell image segmentation. Pattern Recognition, 33(5), 821-832. doi:10.1016/ S0031-3203(99)00091-6

Gonzalez, R., C., Richard E.,W. (2008). Digital Image Processing. Pearson Education, Inc. and Dorling Kindersley Publications, Inc, 2008.

Hou, Z., Hu, Q., \& Nowinsk, W. L. (2006). On minimum variance thresholding. Pattern Recognition Letters, 27(14), 1732-1743. doi:10.1016/j. patrec.2006.04.012

Lassouaoui, N., \& Hamami, L. (2003). Genetic algorithms and multifractal segmentation of cervical cell images. in Proc. 7th Int. Symp. Signal Process. Appl., vol. 2,(pp. 1-4). doi:10.1109/ISSPA.2003.1224800

Li,Z., \& Najarian, K. (2007). Biomedical image segmentation based on shape stability. In: Proceedings of the 14th IEEE International Conference on Image Process (ICIP 2007), San Antonio, (pp 281-284). doi:10.1109/ICIP.2007.4379576

Lin, C. H., Chan, Y. K., \& Chen, C. C. (2009). Detection and segmentation of cervical cell cytoplast and nucleus. International Journal of Imaging Systems and Technology, 19(3), 260-270. doi:10.1002/ ima.20198

Malm, P., \& Brun, A. (2009) Closing curves with Riemannian dilation: application to segmentation in automated cervical cancer screening. In: Proc 5th International Symposium on Visual Computing 2009, Lecture Notes in Computer Science 5875:337-346. doi:10.1007/978-3-642-10331-5 32

Mat Isa, N. A. (2005). Automated edge detection technique for Pap smear images usingmovingKmeans clustering and modified seed based region growing algorithm. Int. J. Comput. Internet Manag., 13(3), 45-59.

Papanicolaou, G. N. (1941). A new procedure for staining vaginal smears. Science, 95(2469), 438-439. doi:10.1126/science.95.2469.438 PMID:17842594 
Plissiti, M. E., \& Nikou, C. (2013). A Review of Automated Techniques for Cervical Cell Image Analysis and Classification. Iacoviello, D. and Andreaus, U. (eds.), Biomedical Imaging and Computational Modeling in Biomechanics, Lecture Notes in Computational Vision and Biomechanics 4, ISSN 2212-9391, ISSN 2212-9413 (electronic), ISBN 978-94-007-4269-7, ISBN 978-94-007-4270-3 (eBook), DOI , Springer Science+Business Media Dordrecht, (pp 1-18).10.1007/978-94-007-4270-3_1

Plissiti, M. E., Nikou, C., \& Charchanti, A. (2010). Accurate localization of cell nuclei in Pap smear images using Gradient Vector Flow deformable models", in Proceedings of the 3rd International Conference on Bio-inspired Signals and Systems (BIOSIGNALS 2010), Valencia, Spain, (pp. 284-289).

Plissiti, M. E., Nikou, C., \& Charchanti, A. (2011a). Automated Detection of Cell Nuclei in Pap Smear Images Using Morphological Reconstruction and Clustering. IEEE Transactions on Information Technology in Biomedicine, 15(2), 233-241. doi:10.1109/ TITB.2010.2087030 PMID:20952343

Plissiti, M. E., Nikou, C., \& Charchanti, A. (2011b). Combining shape, texture and intensity features for cell nuclei extraction in Pap smear images. Pattern Recognition Letters, 32(6), 838-853. doi:10.1016/j. patrec.2011.01.008

Poulsen, R. S., \& Pedron, I. (1995). Region of interest finding in reduced resolution colour imagery application to cancer cell detection. Pattern Recognition, 28(11), 1645-1655. doi:10.1016/00313203(95)00040-7

Riana, D., Dewi, D. E. O., Widyantoro, D. H., \& Mengko, T. L. R. (2014). Color Canals Modification with Canny Edge Detection and Morphological Reconstruction for Cell Nucleus Segmentation and Area Measurement in Normal Pap Smear Images. AIP Conference Proceedings, 1589, 441-447. doi: $10.1063 / 1.4868832$

Sezgin, M., \& Sanlur, B. (2004). Survey over image thresholding techniques and quantitative performance evaluation. Electron. Imag., 13(1), 146-165. doi: $10.1117 / 1.1631315$
Sezgin, M., \& Sanlur, B. (2004). Survey over image thresholding techniques and quantitative performance evaluation. Electron. Imag., 13(1), 146-165. doi:10.1117/1.1631315

Smith, R.A., Baptiste, D. M., Brooks, D., Cokkinides, V., Doroshenk, M., \& Saslow, D. (2014). Cancer Screening in the United States, 2014: A Review of Current American Cancer Society Guidelines and Current Issues in Cancer Screening. CA: a Cancer Journal for Clinicians, 64(1), 30-51. doi:10.3322/ caac.21212 PMID:24408568

Tsai, M. H., Chan, Y. K., Lin, Z. Z., Yang-Mao, S. F., \& Huang, P. C. (2008). Nucleus and cytoplast contour detector of cervical smear image. Pattern Recognition Letters, 29(9), 1441-1453. doi:10.1016/j. patrec.2008.02.024

Tsai, M. H., Wang, M. H., Chang, T. Y., Pai, P. Y., Chan, Y. K., \& Chen, J. M. (2010). An adaptable threshold decision method. International Journal of Innovative Computing, Information, \& Control, $6,2285-2300$.

Wu, H. S., Gil, J., \& Barba, J. (1998) Optimal segmentation of cell images. In: Proc IEE Vision, Image and Signal Process, 145(1), 50-56

Yang-Mao, S. F., Chan, Y. K., \& Chu, Y. P. (2008). Edge enhancement nucleus and cytoplast contour detector of cervical smear images. IEEE Transactions on Systems, Man, and Cybernetics. Part B, Cybernetics, 38(2), 353-366. doi:10.1109/TSMCB.2007.912940 PMID: 18348920

Yung, F. C., Po-Chi, H., Ker-Cheng, L., Hsuan-Hung, L., Li-En, W., Chung-Chuan, C., \& John, Y.C. (2014). Semi-automatic segmentation and classification of pap smear cells. IEEE Journal of Biomedical and Health Informatics, 18(1), 94-108. doi:10.1109/ JBHI.2013.2250984 PMID:24403407 